\title{
Research Paper: Investigation of Gait Initiation in Women With Multiple Sclerosis (MS)
}

\author{
Tayebeh Soleimani $^{1^{*}}$ (D), Heidar Sadeghi ${ }^{2}$ (D), Ali Abbasi² ${ }^{\text {(D) }}$, Saeed Ilbeigi ${ }^{3}$ (D),Farhad Tabatabaei Ghomshe ${ }^{4}$ (C) \\ 1. Faculty of Physical Education And Sport Science, Islamic Azad University of Gonabad Branch, Gonabad, Iran \\ 2. Faculty of Physical Education And Sport Sciences, Kharazmi University, Teheran, Iran. \\ 3. Faculty of Sport Sciences, University of Birjand, Iran. \\ 4. Faculty of Ergonomics, Rehabilitation University, Tehran, Iran.
}

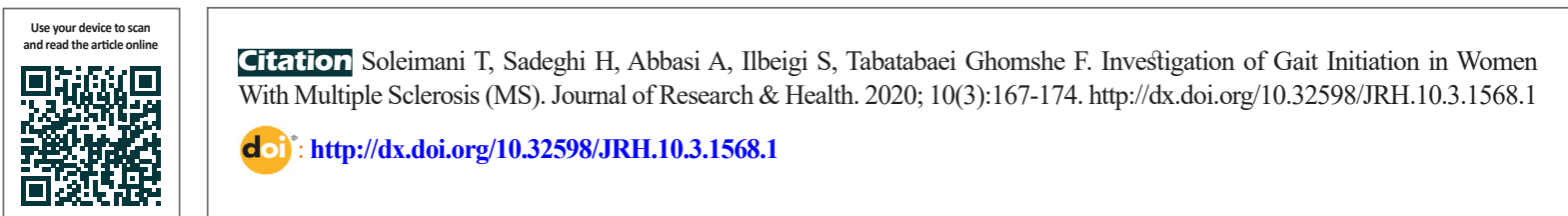

\section{(i) (\$)}

\section{Article info:}

Received: 2 Jul 2018 Accepted: 24 Sep 2018

Publish: 01 May 2020

\section{Keywords:}

Gait analysis, Multiple Sclerosis (MS), Balance

\section{A B S T R A C T}

Background: Walking impairment is one of the most reported symptoms of Multiple Sclerosis (MS). The documentation of gait impairments is important for indexing disease progression and rehabilitation in MS. By measuring and comparing relevant parameters based on the Center of Pressure (COP) patterns, this study aimed to characterize the execution of the gait task and to identify the relationship between these parameters and the level of Expanded Disability Status Scale (EDSS)

Methods: Ten women with MS, with an average EDSS score of 2.4, and 10 healthy women were tested using a force plate and motion capture system during the transition from standing to gait posture. The time series of $\mathrm{COP}$ were acquired and processed to extract the trajectory-related parameters followed by the COP.

Results: The correlation analysis underlined that the progressive alteration of the task execution is directly related to the increase in the EDSS score. These findings suggest that most of the impairments found in people with MS originate from the first part of the COP pattern: the anticipatory postural adjustments (APAs). Before every voluntary movement, the central nervous system performs APAs to minimize the balance perturbation due to the movement itself.

Conclusion: Gait initiation APAs play a role in some contractions of ankle muscles and induce a backward COP shift to the swing limb. Our analysis highlighted that patients who affected by MS have a reduced posterior COP shift that reveals the impairment of the anticipatory mechanism.

\footnotetext{
* Corresponding Author:

Tayebeh Soleimani, MD.

Address: Faculty of Physical Education And Sport Science, Islamic Azad University of Gonabad Branch, Gonabad, Iran.

Phone: +98 (915) 1309203

E-mail: ti_soleimani@yahoo.com
} 


\section{Introduction}

ultiple Sclerosis (MS), as a chronic disease of the Central Nervous Sys$\mathbf{M}$ tem (CNS), affects about 2.2 million people worldwide [1]. MS involves myelin elimination, axonal destruction, and CNS scarring. The CNS deformed structure disrupts the regulation of action potential. These effects are manifested in a variety of symptoms, including, but not limited to, impaired perception, weight gain, and movement dysfunction. Seventy percent of the people with MS report a defect in walking, which is the most challenging aspect of their disease. Half of the people with MS need supportive measures to move through 15 years since the initiation of disease $[2,3]$.

MS leads to advanced disability that is clinically classified by the Extended Disability Status Scale (EDSS) [4]. The EDSS is the most commonly used method for estimating disability in MS. This scale is a neural quantification check scale consisting of 7 Functional Systems (FSs). Each FS subset ranges from 0-5 or 0-6. The EDSS global scale is a combination of the single FS values of an outpatient or autonomous patient in daily activities; this scale ranges from 0 (disability) to 10 (MS death) [5]. The imbalance is associated with the dysfunction of several CNS areas related to balance and gait control, such as the brainstem, spinal cord, and cerebellum [6]. The dysfunction of these areas affects sensory-conduction velocity; pyramidal, vestibular (atrial), and visual impairment; and reduces central integration [7].

Walking is a complex process that involves the coordination of the different systems of the body (e.g., central nerves, skeletal muscles, and cardiovascular system). The individual's nervous system signals to control a large number of muscles and to monitor and correct the movements; it maintains a standing posture [8]. Changes in gait are caused by a combination of factors, because of the multiplicity of muscles and the involved nervous process [9]. Given that the gait impairment is one of the most common symptoms of MS and affects the quality of life, the walking difficulty is a major symptom of MS. Gait impairment is important for indexing disease progression and rehabilitation in MS [10].

Although studying the stationary position is important for analyzing the balance problems, more challenging situations such as Gait Initiation (GI) may provide a deeper insight into the underlying mechanisms of dynamic postural control. The gait change is a complex postural control, as it involves a change from a relatively constant posture [7]. During this postural shift, the controlled muscle effort is needed to move the body's Center of Mass (COM) from the steady-state position, where the COM and the Center of Pressure (COP) are aligned. Thus, gait change leads to inherent instability. In this process, the stability is more affected because the broad base provided by dual support is replaced by a narrower and more unstable state $[6,7]$. The GI plays a key role in moving forward [11-15] and postural stability [16, 17] Therefore, GI represents a natural task (not a deliberate destabilizing task) and can be a valid tool for assessing the ability of the subjects with neurological and movement disorders, including MS.

The GI postural phase is commonly known as the Anticipatory Postural Adjustment (APA) that occurs before the coarse segmental motion phase [18] and supports the first step changes (LOC). The COP changes dynamically at the stability boundary while demonstrating the synergistic behavior of APA. The concerted actions of the two postural mechanisms (ankle and thigh strategies) initiate a set of the systematic change of COP during APA: first, lateral shift to the first swinging heel $\left(\mathrm{APA}_{1}\right)$ and then, the lateral transfer of COP to the foot position leading to a point in the COP that changes from lateral to anterior motion $\left(\mathrm{APA}_{2}\right)$. The third section (LOC) extends from the last point to the first standing foot claw that was being removed from the ground as the COP moves in the anterior-posterior direction $[19,20]$.

Some of the previous studies have evaluated the GI in small groups of MS patients. Remelius et al. examined the GI in women with MS, by calculating temporal and spatial parameters related to the COM and COP displacement [19]. This study shows that the MS group has a smaller, slower, and the shorter distance of the boundary of mass during the GI. The present study differs from the previous studies in the type of test center and the pressure center evaluation protocols that use inverse dynamics [20]. Jacobs and Kasser examined GI in 13 patients with MS. They showed a delayed onset of leg lift and a longer APA compared with the control group. The researchers also emphasized the latency onset of the posterior component of the APA in the dual-task (cognitive+motor) versus the monolithic (motor) condition [21].

According to these data, GI can be an ideal task for identifying abnormalities in the dynamic postural control system and the changes that occur with advancing disability. Considering a wide range of disabilities, this study aimed to determine the kinematics of GI in a group 
of women with MS and to provide a wide range of the involvement of the functional system.

\section{Methods}

The present study is a quasi-experimental study that included 10 women with MS with the Mean \pm SD of age $38.8 \pm 9.5$ (ranged 22-67 years) and 10 healthy women with the Mean \pm SD of age 35.9 \pm 13.1 (ranged 22-71 years) as the healthy control (HC) group. The MS and $\mathrm{HC}$ groups were matched in terms of anthropometric characteristics and age. The inclusion criteria included the diagnosis of MS by a neurologist and the ability to walk without any assistance for at least $2 \mathrm{~m}$. The exclusion criterion was the presence of any concomitant conditions affecting this assessment (i.e, orthopedic or nonMS balance problems). The process of diagnosis lasted 10 days. The EDSS score for MS patients was between 1 and 7 (59\% with a score of 1 to 2, 18\% between 3 and $4,11 \%$ between 4 and 5, 11\% between 5 and 7, and $1 \%$ with a score of 7). The Mean \pm SD of EDSS score was $2.44 \pm 1.64$

When entering the test, participants received an oral explanation about the study procedures and gave their informed consent. The participants were allowed to ask questions. They provided self-report demographic information, including age, gender, the use of assistive devices, the type of MS, and the duration of diagnosis. Then, they performed several gait initiation trials; physiological fall risk tests were also performed.

To measure kinematic and kinetic data, respectively, w used the Qualysis kinematic analysis system consisted of 8 cameras (Qualysis, Switzerland) and the Kistler force plate (Kistler, Switzerland) $(500 \mathrm{~mm} \times 600 \mathrm{~mm}$, model SA 960), which are located in the Health Center of the Islamic Azad University of Mashhad Branch. The system is capable to shoot up to 900 frames per second in $3 \mathrm{D}$. The frequency of the cameras was set at $120 \mathrm{~Hz}$. The cameras were positioned so that each marker could be seen at least by two cameras at any given moment. The cameras were calibrated in such a way that the $\mathrm{x}$ axis was positioned along the trajectory and they were perpendicular to the subjects' frontal plane. The calibration was sufficient to fully cover the subject.

To install passive reflective markers, the subjects were asked to wear special tights, then, 21 markers were mounted on the desired anatomical points. These points included the thumb, toe, heel, medial malleolus ankle, lateral malleolus ankle, tibial prominence, large trochanter of femur, iliac crest in the right and left foot, and 4 markers on the leg and thigh segment. The markers were fixed onto the subjects' body using double-sided adhesive Cushion (to prevent displacement during testing).

Initially, the position of the markers of the subjects was filmed statically; then, the subjects were allowed to adjust to the path they needed to reach. After announcing the subject's readiness and recognizing the examiner, the subjects were asked to stand on the force plate and to place their feet at a comfortable distance. Then, they were instructed to start walking at their chosen speed after verbal input; the auditory signaling device was being in tune with the motion analysis system. The participants were to maintain their posture without moving during the initial stance and to perform the GI movement of their choice. Five walking tests were performed on each subject. The time interval between the signal presentation and the first toe removal was recorded for each test. Also, the walking time averages during trials were calculated.

\section{GI data processing and parameters}

Megawin software enables the acquisition, collection, and the recording of raw COP time series, forces, and torques, which were then processed using a specific procedure in the smart analyzer environment. This procedure facilitates the calculation of quantitative parameters (Figure 1):

a. Mid-lateral and the anterior-posterior coordinates of points A, B, C, and D (Figure 1);

b. The path length $(\mathrm{m})$ of the sections $\mathrm{APA}_{1}, \mathrm{APA}_{2 \mathrm{a}}$ $\mathrm{APA}_{2 \mathrm{~b}}$, and LOC;

c. The mean value of route velocity, regarding midlateral and anterior-posterior displacement $(\mathrm{m} / \mathrm{s})$ in sections $\mathrm{APA}_{1}, \mathrm{APA}_{2 \mathrm{a}}, \mathrm{APA}_{2 \mathrm{~b}}$, and LOC;

$\mathrm{d}$ And the duration of route $(\mathrm{s})$ in sections $\mathrm{APA}_{1}, \mathrm{APA}_{2 \mathrm{a}}$, $\mathrm{APA}_{2 \mathrm{~b}}$, and LOC.

Specifically, the APA and LOC stages were defined based on the studies by Remelius et al. (2008) [19] and Halliday et al. (1998) [18]. To provide a better illustration of this pattern, the APA2 segment (which represents the phase of the weight-to-foot oscillation of the standing leg) was later divided into $\mathrm{APA}_{2 \mathrm{a}}$ and $\mathrm{APA}_{2 \mathrm{~b}}$ (Figure $1)$.

After examining the normality, the mean values of Q1 and Q3 were calculated for the parameters of the two 


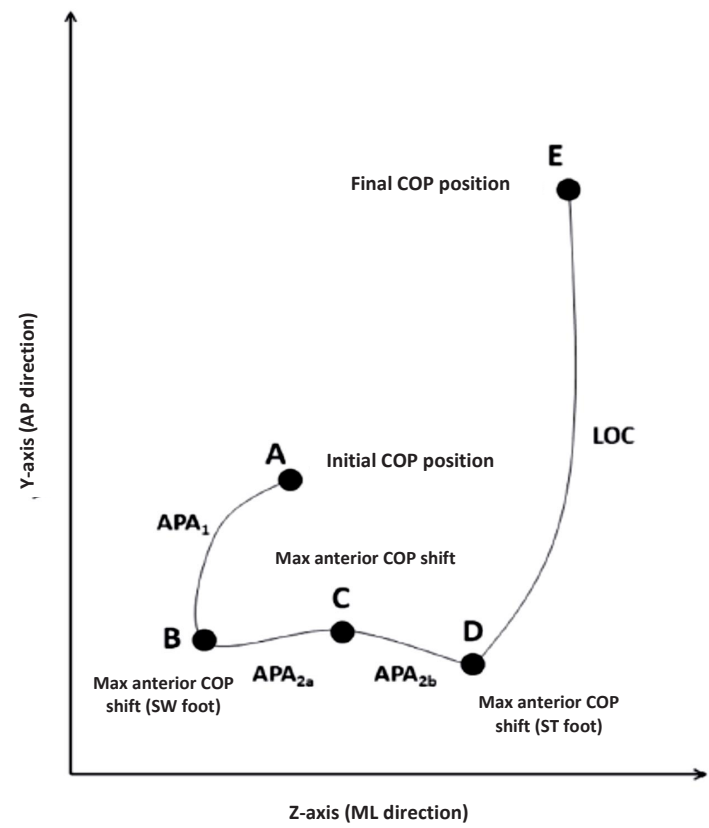

Figure 1. The COP route split diagram of analysis $\left(\mathrm{APA}_{1^{\prime}}, \mathrm{APA}_{2 \mathrm{a}^{\prime}} \mathrm{APA}_{2 \mathrm{~b}^{\prime}}\right.$ and LOC)

SW: swinging foot (i.e., front foot); ST: standing foot (i.e., footrest in GI).

groups using the Kolmogorov-Smirnov test. Betweengroup differences were analyzed using the Mann-Whitney $\mathrm{U}$ test. The significance level was set at $\mathrm{P}<0.05$. The relationships between the EDSS score and GI parameters were evaluated using the Spearman rank correlation analysis.

\section{Results}

The position of the legs did not differ in the starting position during the subjects' evaluation. Among the whole set of parameters, 6 cases (listed in Table 1) caused a significant difference between the MS and $\mathrm{HC}$ groups.
Table 2 reports the results of the correlation analysis. The EDSS score is positively correlated with the $\mathrm{Y}$ coordinate of point $\mathrm{B}(\mathrm{P}<0.001, \rho=0.513)$, the $\mathrm{Y}$ component of the APA velocity $(\mathrm{P}<0.008, \rho=0.264)$, the duration of $\mathrm{APA}_{2 \mathrm{~b}}(\mathrm{P}<0.001, \rho=0.423)$, and the duration of LOC $(\mathrm{P}<0.001, \rho=0.475)$. Also, the EDSS score is negatively correlated with the $\mathrm{Y}$ component of $\mathrm{APA}_{2 \mathrm{a}}$ velocity $(\mathrm{P}<0.039, \rho=-0.218)$, and the $\mathrm{Y}$ component of LOC velocity $(\mathrm{P}<0.001, \rho=-0.284)$.

\section{Discussion}

Table 1. Mean, Median, Q1, and Q3 values for statistically significant parameters after running the Mann-Whitney Test in MS and $\mathrm{HC}$ groups

\begin{tabular}{|c|c|c|c|c|c|c|c|c|}
\hline \multicolumn{9}{|c|}{ GI Parameters } \\
\hline \multirow{2}{*}{ Parameter } & \multicolumn{4}{|c|}{ MS Group } & \multicolumn{4}{|c|}{ HC Group } \\
\hline & Mean & Median & Q1 & Q3 & Mean & Median & Q1 & Q3 \\
\hline$Y$ coordinate of point $B(m)$ & -0.022 & -0.019 & -0.032 & -0.009 & -0.034 & -0.029 & -0.042 & -0.042 \\
\hline $\begin{array}{l}\text { Y component of the } \mathrm{APA}_{1} \text { veloc- } \\
\text { ity }(\mathrm{m} / \mathrm{s})\end{array}$ & -0.017 & -0.015 & -0.023 & -0.006 & -0.024 & 0.022 & -0.027 & -0.015 \\
\hline $\begin{array}{l}\text { Y component of the } A A_{2 a} \\
\text { velocity }(\mathrm{m} / \mathrm{s})\end{array}$ & 0.112 & 0.109 & 0.039 & 0.171 & 0.150 & 0.146 & 0.102 & 0.184 \\
\hline $\begin{array}{l}\text { Y component of LOC velocity } \\
\qquad(\mathrm{m} / \mathrm{s})\end{array}$ & 0.233 & 0.237 & 0.180 & 0.289 & 0.290 & 0.286 & 0.226 & 0.343 \\
\hline $\mathrm{APA}_{2 \mathrm{~b}}$ Duration (s) & 0.140 & 0.650 & 0.575 & 0.820 & 0.609 & 0.590 & 0.525 & 0.660 \\
\hline Duration LOC (s) & 0.802 & 0.650 & 0.575 & 0.820 & 0.609 & 0.590 & 0.525 & 0.660 \\
\hline
\end{tabular}

IMHA 
Table 2. Results of Correlation Analysis for EDSS score and GI parameters

\begin{tabular}{cccc}
\hline Variables & No. of Valid & $\rho$ & P \\
\hline Y coordinate of point B - EDSS & 93 & 0.513 & 0.001 \\
\hline Y component of the APA $A_{1}$ velocity - EDSS & 93 & 0.264 & 0.008 \\
Y component of the APA 2 velocity - EDSS & 84 & -0.218 & 0.039 \\
Y component of LOC velocity - EDSS & 88 & -0.284 & 0.001 \\
\hline EDSS - APA 2 Duration & 84 & 0.423 & 0.001 \\
\hline EDSS - Duration LOC & 88 & 0.475 & 0.001 \\
\hline
\end{tabular}

URA

According to the present results, the main feature of GI in MS patients is the reduction of the speed and the displacement of posterior COP. These findings confirm the findings of previous studies on MS $[22,23]$. It is important to note that the speed significantly decreased at $\mathrm{APA}_{1}$ and $\mathrm{APA}_{2 \mathrm{a}}$. These sections comprise the early phase of APAs and are consecutive for most of the posterior COP rotations (point $\mathrm{B}$ ). The velocity in the $\mathrm{Y}$ direction and the displacement of point B appear differently: preventing the stability boundary in the posterior direction at initial APA lengths.

At the LOC stage, the COP speed is reduced due to several reasons. According to APAs, there is a functional adaptation aimed at avoiding sustained perturbations. Moreover, the power reduction can play a major role in reducing the moving speed and the slowing of the COP movement. Also, the LOC stage mainly tends to the Ydirection, and this tendency may result in significant changes in the anterior-posterior component.

Posterior displacement of point B significantly decreased in patients, compared with the HC group. These findings are consistent with the results of Remelius et al. and indicate the postural damage in MS along with other pathological conditions [19].

The backward direction of COP is changed during APAs to move the COM forward and to reach the desired walking speed at the end of step one [11-15]. Therefore, the reduction of point $\mathrm{B}$ shows a strategy that reduces the forward thrust at COM and LOC speeds to make the walking safer. According to these data, the reduction of the posterior displacement of point B in MS patients can be interpreted not only as movement planning but also as a competitive strategy. This fact indicates functional compatibility that aimed to minimize these posterior levels of stability and to decrease the risk of falling. Also, at point $\mathrm{B}, \mathrm{COP}$ has a strong circulation across the GI, particularly near the stability boundaries [20].

These findings emphasize that the kinetic parameters of GI indicate the patterns of MS disability. Particularly, the $\mathrm{Y}$ coordinates of point B are correlated with EDSS. Thus, the posterior displacement of COP in the early phase of APA is closely related to postural disorder and disability. The $\mathrm{Y}$ coordinate of point $\mathrm{B}$ is a suitable alternative to postural disorder and can be used as an indicator of the progression of disability and the risk of falls, also, it is useful in evaluating rehabilitation.

Previous studies in patients with Parkinson disease have shown a relationship between APA perturbations and the injury of the areas involved in movement planning. In particular, the relationship between the basal ganglia and the complementary and prefrontal regions or the cerebellum is involved [24, 25].

This issue cannot be ignored in the context of the widespread damage of white and gray matter, which contributes to the specific APA pattern in MS. Besides, as shown by other authors [6], because of the spread of CNS damage, different stimuli can play a major role in the postural disorder of MS.

\section{Conclusion}

The properties of the COP pathway show a pattern of changes that overlap with other neurological pathologies. The posterior displacement of COP in the early phase of APA can play an important role in the measurement of the postural disorder. Also, this study could lead to the COP-focused rehabilitation strategies aimed at the retraining of MS patients for the use of proper positionto-movement transition. 


\section{Ethical Considerations}

\section{Compliance with ethical guidelines}

All ethical principles were considered in this article.

Funding

This article is a part of a $\mathrm{PhD}$ dissertation in the Department of Sport Biomechanics at Kharazmi University of Tehran, Iran by Tayebeh soleimani.

\section{Authors' contributions}

Conceptualization, writing - original draft: Tayebeh Soleimani , Heidar Sadeghi, Saeed Ilbeigi, Farhad Tabatabaei Ghomshe; Methodology: Tayebeh Soleimani , Farhad Tabatabaei Ghomshe; Investigation: Tayebeh Soleimani , Heidar Sadeghi, Saeed Ilbeigi; Writing review \& editing: Tayebeh Soleimani, Heidar Sadeghi, Ali Abbasi; Funding acquisition: Tayebeh Soleimani , Heidar Sadeghi; Resources: Tayebeh Soleimani, Farhad Tabatabaei Ghomshe; Supervision: Ali Abbasi, Heidar Sadeghi, Saeed Ilbeigi, Farhad Tabatabaei Ghomshe.

\section{Conflict of interest}

The authors declared no conflict of interest.

\section{Refrences}

[1] Kalb R, Reitman N. Multiple sclerosis: A model of psychological support. $5^{\text {th }}$ edition. National Multiple Sclerosis Society; 2012. https://www.nationalmssociety.org/NationalMSSociety/ media/MSNationalFiles/Brochures/Psychosocial-Cavallo-5thEdition_Final-Links.pdf

[2] Noseworthy JH, Lucchinetti C, Rodriguez M, Weinshenker BG. Multiple sclerosis. N Engl J Med. 2000; 343:938-52. [DOI:10.1056/NEJM200009283431307] [PMID]

[3] Pearson OR, Busse ME, van Deursen RWM, Wiles CM Quantification of walking mobility in neurological disorders. QJM: An International Journal of Medicine. 2004; 97(8):46375. [DOI:10.1093/qjmed/hch084] [PMID]

[4] Kurtzke, JF. Rating neurologic impairment in multiple sclerosis: An expanded disability status scale (EDSS). Neurol. 1983; 33(11):1444-52. [DOI:10.1212/WNL.33.11.1444] [PMID]

[5] Martin CL, Phillips BA, Kilpatrick TJ, Butzkueven H, Tubridy $\mathrm{N}, \mathrm{McDonald} \mathrm{E}$, et al. Gait and balance impairment in early Multiple Sclerosis in the absence of clinical disability. Mult Scler J. 2006; 12(5):620-8. [DOI:10.1177/1352458506070658] [PMID]
[6] Martin M, Shinberg M, Kuchibhatla M, Ray L, Carollo JJ Schenkman ML. Gait initiation in community dwelling adults with Parkinson disease: Comparison with older and younger adults without the disease. Phys Ther. 2002; 82(6):566-77. [DOI:10.1093/ptj/82.6.566] [PMID]

[7] Winter DA. Biomechanics and motor control of human movement. Hoboken: John Wiley \& Sons; 2009. [DOI:10.1002/9780470549148]

[8] Callisaya ML, Blizzard L, McGinley JL, Schmidt MD, Srikanth VK. Sensorimotor factors affecting gait variability in older people -- a population-based study. J Gerontol Ser. 2010; 65(4):386-92. [DOI:10.1093/gerona/glp184] [PMID]

[9] LaRocca NG. Impact of walking impairment in multiple sclerosis: Perspectives of patients and care partners. Patient. 2011; 4(3):189-201. [DOI:10.2165/11591150-000000000-00000] [PMID]

[10] Brunt D, Liu SM, Trimble M, Bauer, J, Short M. Principles underlying the organization of movement initiation from quiet stance. Gait \& Posture. 1999; 10(2):121-8. [DOI:10.1016/ S0966-6362(99)00020-X]

[11] Brenière $Y$, Do MC, Bouisset $S$. Are dynamic phenomen a prior to stepping essential to walking? J Motor Behav. 1987; 19(1):62-76. [DOI:10.1080/00222895.1987.10735400] [PMID]

[12] Couillandre A, Brenière $Y$, Maton B. Is human gait initiation program affected by a reduction of the postural basis? Neurosci Lett. 2000; 285(2):150-4. [DOI:10.1016/S03043940(00)01015-6]

[13] Gélat T, Pellec AL, Brenière Y. Evidence for a common processing gait initiation and stepping on to a new level to reach gait velocity. Exp Brain Res. 2006; 170(3):336-44. [DOI:10.1007/ s00221-005-0214-8] [PMID]

[14] Lepers R, Brenière $Y$. The role of anticipatory postural adjustments and gravity in gait initiation. Exp Brain Res. 1995 107(1):118-24. [DOI:10.1007/BF00228023] [PMID]

[15] Caderby T, Yiou E, Peyrot N, Begon M, Dalleau G. Influence of gait speed on the control of mediolateral dynamic stability during gait initiation. J Biomech. 2014; 47(2):417-23. [DOI:10.1016/j.jbiomech.2013.11.011] [PMID]

[16] McIlroy WE, Maki BE. The control of lateral stability during rapid stepping reactions evoked by antero-posterior perturbation: Does anticipatory control play a role? Gait \& Posture. 1999; 9(3):190-8. [DOI:10.1016/S0966-6362(99)00013-2]

[17] Brenière $Y$, Do $M$. When and how does steady state gait movement induced from upright posture begin? J Biomech. 1986; 19(12):1035-40. [DOI:10.1016/0021-9290(86)90120-X]

[18] Halliday SE, Winter DA, Frank JS, Patla AE, Prince F. 1998. The initiation of gait in young, elderly, and Parkinson's disease subjects. Gait \& Posture. 1998; 8(1):8-14. [DOI:10.1016/ S0966-6362(98)00020-4]

[19] Remelius JG, Hamill J, Kent-Braun J, Van Emmerik RE. Gait initiation in multiple sclerosis. Motor Control. 2008; 12(2):93-108. [DOI:10.1123/mcj.12.2.93] [PMID]

[20] Jacobs J, Kasser S. Effects of dual tasking on the postural performance of people with and without multiple sclerosis: A pilot study. J Neurol. 2012; 259:1166-76. [DOI:10.1007/s00415011-6321-5] [PMID] 
[21] Mbourou GA, Lajoie Y, Teasdale N. Step length variability at gait initiation in elderly fallers and non-fallers, and young adults. Gerontol. 2003; 49(1):21-6. [DOI:10.1159/000066506] [PMID]

[22] Prosperini L, Kouleridou A, Petsas N, Leonardi L, Tona $\mathrm{F}$, Pantano $\mathrm{P}$, et al. The relationship between infratentorial lesions, balance deficit and accidental falls in multiple sclerosis. J Neurol Sci. 2011; 304(1-2):55-60. [DOI:10.1016/j. jns.2011.02.014] [PMID]

[23] Massion J. Movement, posture and equilibrium: Interaction and coordination. Prog Neurobiol. 1992; 38(1):35-56. [DOI:10.1016/0301-0082(92)90034-C]

[24] Nakano K, Kayahara T, Tsutsumi T, Ushiro H. Neural circuits and functional organization of the striatum. J Neurol 2000; 247(Suppl. 5):V1-15. [DOI:10.1007/PL00007778] [PMID]

[25] Pahapil PA, Lozano AM. The pedunculopontine nucleus and Parkinson's disease. Brain. 2000; 123(Pt 9):1767-83. [DOI:10.1093/brain/123.9.1767] [PMID] 
This Page Intentionally Left Blank 